\title{
Impact of perioperative intravenous lidocaine infusion on postoperative pain and rapid recovery of patients undergoing gastrointestinal tumor surgery: a randomized, double-blind trial
}

\author{
Yue'e Dai ${ }^{1,2}$, Rong Jiang', Wenjie $\mathrm{Su}^{2}$, Man Wang ${ }^{2}$, Yue Liu ${ }^{2}$, Yunxia Zuo ${ }^{1}$ \\ ${ }^{1}$ Department of Anesthesiology, West China Hospital, Sichuan University, Chengdu, China; ${ }^{2}$ Department of Anesthesiology, Sichuan Academy of \\ Medical Sciences and Sichuan Provincial People's Hospital, University of Electronic Science and Technology of China, Chengdu, China \\ Contributions: (I) Conception and design: Y Dai; (II) Administrative support: R Jiang, W Su; (III) Provision of study materials or patients: R Jiang; \\ (IV) Collection and assembly of data: M Wang; (V) Data analysis and interpretation: Y Dai, Y Zuo; (VI) Manuscript writing: All authors; (VII) Final \\ approval of manuscript: All authors. \\ Correspondence to: Yunxia Zuo, MD, PhD. Department of Anesthesiology, West China Hospital, Sichuan University, No. 37 Guoxue Xiang, Chengdu \\ 610041, China. Email: zuoyunxia@scu.edu.cn.
}

Background: To explore the effect of perioperative intravenous lidocaine infusion on postoperative pain and the rapid recovery of patients undergoing gastrointestinal tumor surgery.

Methods: The patients who underwent gastrointestinal tumor surgery from May to July 2020 were selected. The patients were randomly divided into the lidocaine group (group L) and control group (group C) by the random number table method, with 60 patients in each group. Both groups of patients received an intravenous drug infusion immediately after induction of tracheal intubation under general anesthesia. In group L, $1.5 \mathrm{mg} / \mathrm{kg}$ lidocaine was slowly injected intravenously at a rate of $1.5 \mathrm{mg} \cdot \mathrm{kg}^{-1} \cdot \mathrm{h}^{-1}$ to the surgical suture, and intravenous inhalation was used to maintain the depth of anesthesia. Group C patients were given the same volume of normal saline. The 2-, 4-, 7-, 14-, 30-, and 90-day numerical rating scale (NRS) and the proportion of chronic post-surgical pain (CPSP) after 3 months for both groups after surgery were recorded. Each patient's postoperative comfort score, requiring analgesia, return of flatus, bowl movement, hospitalization days, hospitalization expenses, and adverse events were also recorded.

Results: One hundred and twenty patients were enrolled but 5 of them failed to complete the treatment process. Therefore, 58 and 57 patients in group $\mathrm{L}$ and $\mathrm{C}$ were included into the final analysis. The NRS of patients in group $\mathrm{L}$ was significantly lower than that of group $\mathrm{C}$ at all time points after surgery $(\mathrm{P}<0.05)$, and the proportion of CPSP in group $\mathrm{L}$ was significantly lower than that of group $\mathrm{C}(\mathrm{P}<0.05)$. The percentage of patients requiring analgesia and postoperative comfort score of group $\mathrm{L}$ was significantly higher than that of group $\mathrm{C}(\mathrm{P}<0.01)$, patient's return of flatus, bowl movement, hospitalization days, and hospitalization expenses in group $\mathrm{L}$ were significantly lower than those in group $\mathrm{C}(\mathrm{P}<0.05)$. There were no difference of adverse events between the 2 groups $(\mathrm{P}>0.05)$.

Conclusions: During the perioperative period of radical gastrointestinal tumor surgery, intravenous lidocaine infusion can reduce acute postoperative pain, promote postoperative gastrointestinal function recovery, and improve postoperative comfort.

Keywords: Intravenous infusion; lidocaine; gastrointestinal tumors; pain; comfort

Submitted Oct 18, 2020. Accepted for publication Dec 08, 2020.

doi: 10.21037/jgo-20-505

View this article at: http://dx.doi.org/10.21037/jgo-20-505 


\section{Introduction}

Radical gastrointestinal tumor resection is currently the main treatment for gastrointestinal tumors. Despite continuous improvements in surgical techniques and perioperative management, the perioperative mortality rate of radical resection of gastrointestinal tumors is still high (1). Among the factors contributing to the low quality of life of patients during the perioperative period, postoperative ileus (POI) is the main influencing factor (2). POI could cause abdominal distension, nausea, pain, and delayed defecation time, and affect intestinal function recovery, prolong hospital stay, and increase economic burden on the patient (3).

Enhanced recovery after surgery (ERAS) and postoperative comfort can also be a good evaluation of the overall medical level. Effective perioperative pain management and the promotion of gastrointestinal function recovery are important for ERAS and postoperative comfort following major gastrointestinal tumor surgery. Opioids are commonly used analgesics during the perioperative period, but they are associated with a risk of slow bowel motility, intestinal paralysis, intestinal obstruction. The dosage of opioids was positively correlated with POI. The greater the dosage was, the longer the POI was (4). Current Clinical treatment to treat POI involves reducing inflammation postoperatively, effective postoperative analgesia but reducing the use of opioids, early oral intake, and early movement. However, there is no single drug or treatment that can effectively manage perioperative pain and gastrointestinal function.

Lidocaine is a short-acting amide local anesthetic, which has anti-inflammatory, analgesic, and promotion of gastrointestinal function recovery. Studies have shown that the use of intravenous lidocaine during open or laparoscopic surgery can reduce the consumption of opioids and other analgesics, reduce the degree of acute postoperative pain in patients, and promote the recovery of gastrointestinal function (5). However, in a meta-analysis, Kranke $e t$ al. questioned the effect of intravenous lidocaine infusion in improving postoperative acute pain (6), and at present, to the best of our knowledge, there is no relevant research on the incidence of chronic post-surgical pain (CPSP) and postoperative comfort after radical resection of gastrointestinal tumors by intravenous lidocaine infusion. The aim of the present study was to investigate the effects of intravenous lidocaine infusion on acute pain, CPSP and postoperative comfort in patients undergoing radical gastrointestinal tumor surgery, and to further evaluate the effectiveness and safety of intravenous lidocaine infusion. We present the following article in accordance with the CONSORT reporting checklist (available at http://dx.doi. org/10.21037/jgo-20-505).

\section{Methods}

The present study was a randomized, double-blind, controlled study. It was reviewed and approved by the Medical Ethics Committee of Sichuan Provincial People's Hospital. All procedures performed in this study involving human participants were in accordance with the Declaration of Helsinki (as revised in 2013). Written informed consent was obtained from patients prior to their participation. A total of 186 patients who had undergone elective radical resection of gastric cancer, colon cancer, and rectal cancer at our hospital from May to July 2020 were assessed for eligibility and 120 patients selected. The random number table method was used to randomly divide the patients into the lidocaine group (group L) and control group (group C), with 60 patients in each group.

\section{Inclusion and exclusion criteria}

The inclusion criteria were as follows: (I) patients undergoing radical resection of gastric cancer, colon cancer, or rectal cancer; (II) aged 20-80 years; (III) American Society of Anaestheiologists (ASA) grades I-II; and (IV) body mass index $18-24 \mathrm{~kg} / \mathrm{m}^{2}$. The exclusion criteria were as follows: (I) patients who were allergic to the lidocaine; (II) blood coagulation dysfunction; (III) a long-term history of opioid or antiemetic use; (IV) patients with severe medical diseases, including high-risk hypertension, a history of myocardial infarction within 3 months, poor diabetes control, severe liver or kidney dysfunction, severe pulmonary heart disease, and atrioventricular block; and (V) those with severe central neuropathy, arrhythmia, and liver or kidney dysfunction, which would make it difficult to accurately assess the effectiveness and safety of the treatment.

\section{Anesthetic and intervention methods}

All patients provided signed informed consent and routinely fasted for $8 \mathrm{~h}$ prior to surgery. Patients' non-invasive blood pressure, heart rate, and oxygen saturation were routinely monitored by electrocardiogram. Mean arterial pressure was measured by radial artery puncture, and central 
venous pressure was measured by internal jugular vein puncture. General anesthetic induction was as follows: $1.5-2 \mathrm{mg} / \mathrm{kg}$ propofol, $0.4 \mu \mathrm{g} / \mathrm{kg}$ sufentanil, and $0.15 \mathrm{mg} / \mathrm{kg}$ cisatracurium. A video laryngoscope was used to assist tracheal intubation for mechanical ventilation. Anesthetic ventilator parameter settings were as follows: oxygen flow rate of $1.5 \mathrm{~L} / \mathrm{min}$, tidal volume of $7-10 \mathrm{~mL} / \mathrm{kg}$, respiratory rate of 10-12 times/min, and end-tidal carbon dioxide partial pressure of $35-45 \mathrm{mmHg}$. The intravenous and inhalation anesthetic were used to maintain the depth of anesthesia at a bispectral index (BIS) value of 40-60. The infusion of general anesthetics was stopped during skin suture. Both groups of patients were given the drug immediately after tracheal intubation. Group L was given a slow intravenous injection of $1.5 \mathrm{mg} / \mathrm{kg}$ lidocaine at a rate of $1.5 \mathrm{mg} \cdot \mathrm{kg}^{-1} \cdot \mathrm{h}^{-1}$ until the surgical suture; group $C$ was given the same volume of normal saline. Both groups were connected to a self-controlled intravenous analgesia pump, and $2 \mu \mathrm{g} / \mathrm{kg}$ sufentanil $+4 \mathrm{mg}$ tropisetron (total $100 \mathrm{~mL}$ ) was administered. Analgesia pump parameter settings were as follows: standard flow rate $2 \mathrm{~mL} / \mathrm{h}$, self-controlled administration dose $1 \mathrm{~mL} /$ time, and lock time of $15 \mathrm{~min}$. Intravenously infusion of drugs was prepared by an nurse and the anesthesiologist did not know the drugs.

\section{Observation index}

The primary observation index in the study were pain and postoperative comfort assessment after surgery. The numerical rating scale (NRS) of both groups at 2, 4, 7, 14,30 , and 90 days postoperatively were recorded. The NRS scores ranged from 0 to 10 points, with 0 points representing no pain and 10 points representing the strongest pain. The pain score was the average of the 2 pain scores in the active and resting states. In all groups at any time, tramadol $100 \mathrm{mg}$ was administered intramuscular as rescue medication if the NRS score was $\geq 7$. The maximum permitted dosage of tramadol was $400 \mathrm{mg}$ per day. To determine patients whether or not had chronic pain postoperatively, patients were followed up by telephone 3 months after the operation and asked if they were still suffering from surgery-related pain. The pain score was evaluated by NRS. In our study, the Quality of Recovery Score-15 (QoR-15) was used to evaluate patients' postoperative comfort before discharge (7). The secondary observation index was postoperative recovery, including the return of flatus, the bowl movement, the hospitalization days. And the hospitalization expenses, the number of analgesic pump compression, and the number of patients on pain medication from the postoperative period to discharge were also observed. The same physician, who was blinded to the trial's groups, recorded adverse events, which included postoperative arrhythmia, nausea, vomiting, headache, dry mouth, and other complications. For each given patient, one or more incidence of the same adverse effect were recorded as one incidence.

\section{Statistical analysis}

SPSS version 17.0 software (SPPS Inc., Cary, NC, USA) was used for the statistical analysis. The measurement data were expressed as mean \pm standard deviation, and the comparison between groups was analyzed by independent samples $t$-test. Count data were expressed as percentage using a 4 -grid table for the $\chi^{2}$-test, and the $U$-test was used to compare grade data. $\mathrm{P}<0.05$ indicated statistical significance.

\section{Results}

\section{Comparison of general information between the 2 groups}

Initially, 186 patients were assessed for the eligibility. Finally, a total of 120 participants were randomized to the two groups, with 60 patients in each group. Two patients in group $\mathrm{L}$ and 3 patients in group $\mathrm{C}$ withdrew due to postoperative follow-up failure, underwent multiple surgeries, or transferred to intensive care unit (ICU) after surgery. Therefore, they were excluded from the study. Finally, 58 patients and 57 patients in group L and C were included. Patient enrollment flow chart was illustrated in Figure 1. No statistically significant difference between the 2 groups in terms of sex, age, height, weight, operation time, lidocaine infusion time, PACU stay time, and type of operation $(\mathrm{P}>0.05)$ (Table 1).

\section{Comparison of postoperative acute and chronic pain and comfort between the 2 groups}

The NRS scores of patients in group $\mathrm{L}$ at 2, 4, 7, 14, 30, and 90 days postoperatively were lower than those in group $\mathrm{C}$ $(\mathrm{P}<0.05)$ (Figure 2). The proportion of patients in group $\mathrm{L}$ who needed additional analgesics to relieve pain from surgery to discharge was significantly lower than that in group $\mathrm{C}$ $(\mathrm{P}<0.01)$ (Figure $3 A)$. The proportion of CPSP in group $\mathrm{L}$ at 3 months postoperatively was lower than that in group $\mathrm{C}$ 


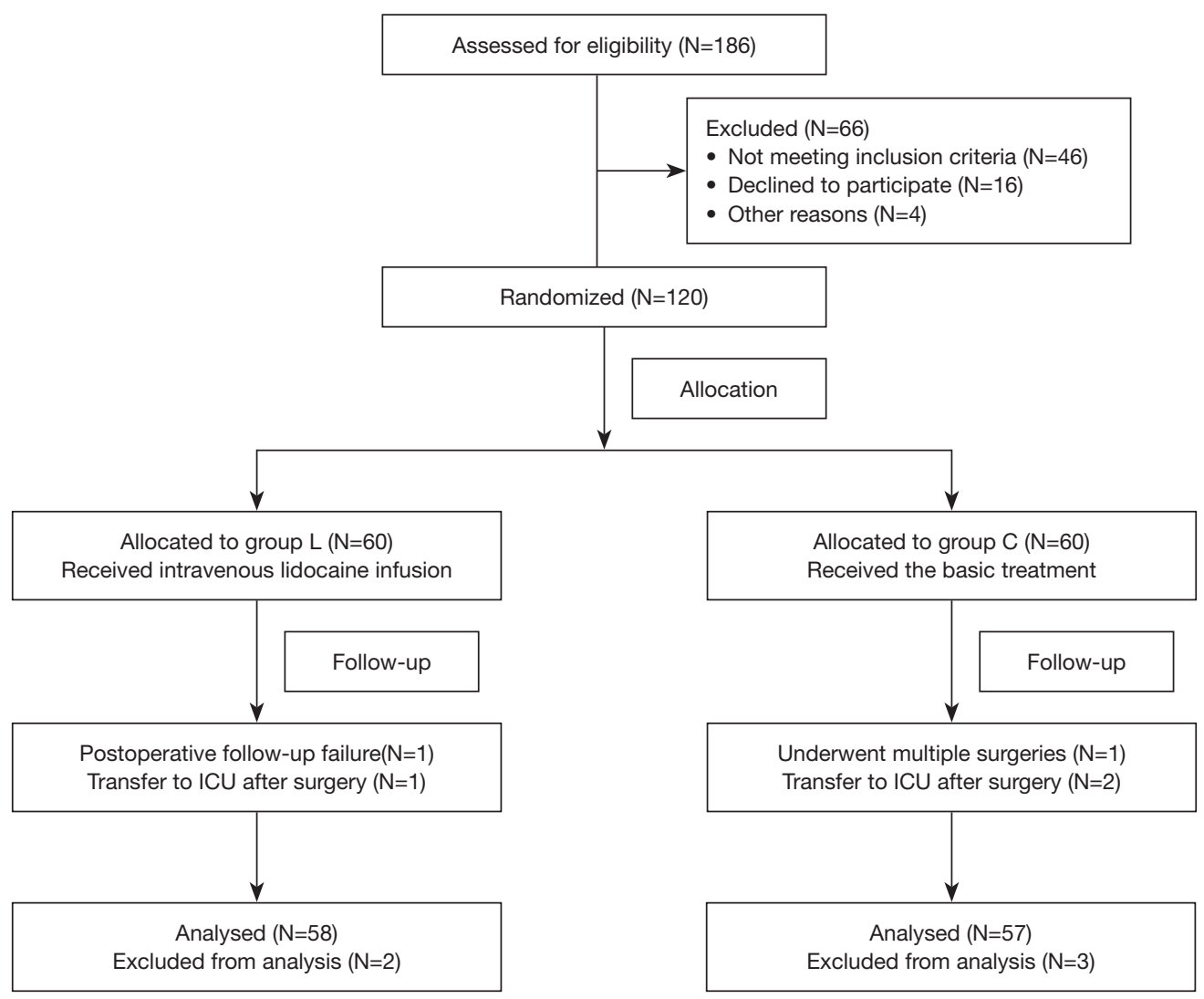

Figure 1 Patient enrollment flow chart.

$(\mathrm{P}<0.05)$ (Figure 3B). Postoperative comfort of group L was significantly higher than that of group $\mathrm{C}$, and the difference was statistically significant $(\mathrm{P}<0.01)$ (Figure 3 C).

\section{Comparison of postoperative recovery between the 2 groups}

The postoperative gastrointestinal recovery index including the return of flatus, the bowl movement in group $\mathrm{L}$ were significantly lower than those in group $\mathrm{C}(\mathrm{P}<0.05$, Figure $4 A$ ). Patients in group $\mathrm{L}$ had significantly lower hospitalization time, and hospitalization expenses than patients in group $\mathrm{C}(\mathrm{P}<0.05$, Figure $4 B, C)$.

\section{Comparison of postoperative adverse events between the 2 groups}

In group L, 7 patients complained of drowsiness and 3 patients complained of dry mouth. In group C, 2 patients complained of dizziness, 5 patients of drowsiness, and 1 patient of nausea and vomiting, but there was no statistical significance $(\mathrm{P}>0.05)$.

\section{Discussion}

The effective perioperative pain management and gastrointestinal function recovery are important for the patients who underwent gastrointestinal tumor surgery. In this study, it was found that intravenous lidocaine infusion significantly improved the postoperative recovery of patients in group L compared with patients in group $\mathrm{C}$, including the effective pain management and the first return of flatus and bowl movement after the operation. It was also observed that intravenous lidocaine infusion can accelerate patients' postoperative recovery, improve their postoperative comfort, and reduce the length of hospitalization and expenses.

Postoperative pain is the primary problem faced by all patients. Lidocaine is an amide local anesthetic that can inhibit the activation of sodium ion channels in nerve cells and block neural pain signals to significantly reduce pain (7). It has also been reported in the literature that intravenous lidocaine infusion can achieve an analgesic effect similar to that of morphine and could reduce the dosage of opioids 
Table 1 Demographic data

\begin{tabular}{lcc}
\hline Indicators & Lidocaine group $(\mathrm{n}=58)$ & Control group $(\mathrm{n}=57)$ \\
\hline Age (years, mean \pm SD) & $61.4 \pm 5.4$ & $63.7 \pm 4.5$ \\
Sex (female/male) & $28 / 30$ & $29 / 28$ \\
Height (cm, mean \pm SD) & $161.7 \pm 7.7$ & $162.5 \pm 8.3$ \\
Weight (kg, mean \pm SD) & $53.8 \pm 7.3$ & $54.3 \pm 6.6$ \\
Surgical time (min, mean \pm SD) & $197.5 \pm 20.4$ & $195.4 \pm 17.8$ \\
Infusion time (min, mean \pm SD) & $163.6 \pm 25.3$ & $164.1 \pm 24.6$ \\
PACU time (min, mean \pm SD) & $80.5 \pm 8.6$ & $78.6 \pm 7.5$ \\
Surgical procedure $(\mathrm{n})$ & & 29 \\
Radical resection of gastric cancer & 26 & 16 \\
Radical resection of colon cancer & 18 & 12 \\
$\quad$ Radical resection of rectal cancer & 14 & \\
\hline
\end{tabular}

PACU, post-anesthesia care unit; SD, standard deviation.

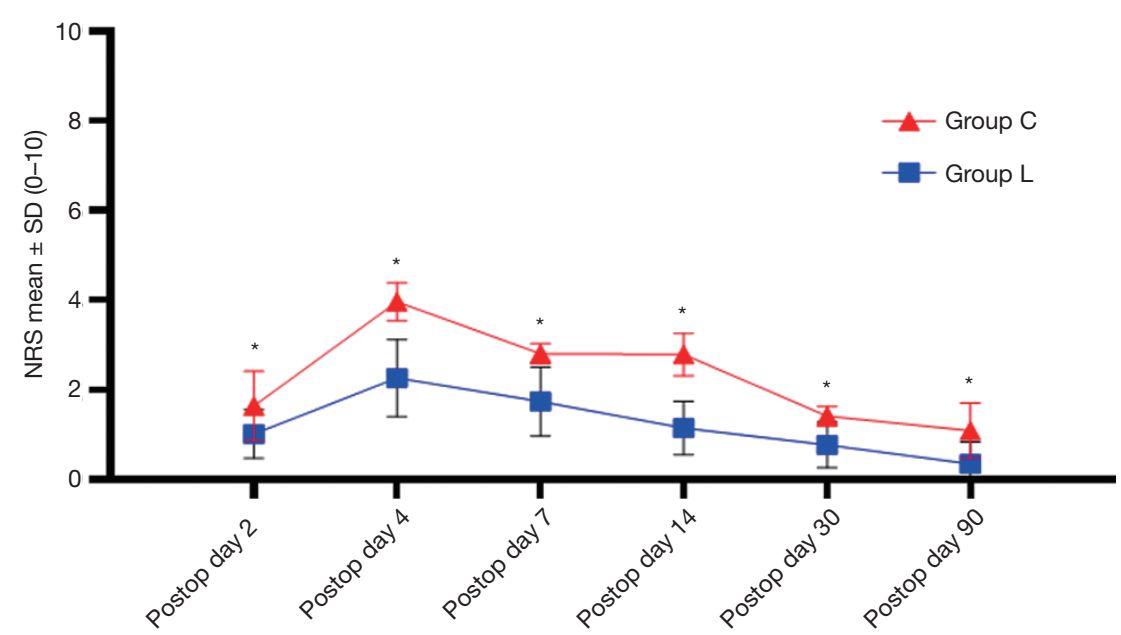

Figure 2 Pain score at different time points after surgery. Numerical rating scale (NRS) scores of the intravenous lidocaine infusion group at different time points after surgery were lower than those of the control group, and the difference was statistically significant $(\mathrm{P}<0.05)$. ${ }^{*} \mathrm{P}<0.05$. SD, standard deviation; Postop, postoperative.

intraoperative (8). Liu et al. reported that intravenous lidocaine infusion can reduce the neuropathic pain caused by herpes zoster and improve mood (9). Moreover, it has been found that intravenous lidocaine has superior efficacy to morphine for renal colic and critical limb ischemia, superior efficacy to dihydroergotamine for acute migraine, and equivalent efficacy to ketorolac for acute radicular lower back pain (10). However, some studies have questioned the analgesic effect of intravenous lidocaine infusion (6). Therefore, the analgesic drugs tramadol was used to rescue the severe pain postoperative by patients were statistically analyzed. Intravenous lidocaine infusion was found to reduce acute postoperative pain and the need for postoperative analgesics in this study.

The impact of intravenous lidocaine infusion on patients with CPSP was also the focus of the present study (11). The 2 groups of patients were followed up for CPSP at 3 months postoperatively. The results showed that intravenous lidocaine infusion can effectively reduce the incidence of CPSP in patients with gastrointestinal tumors, 

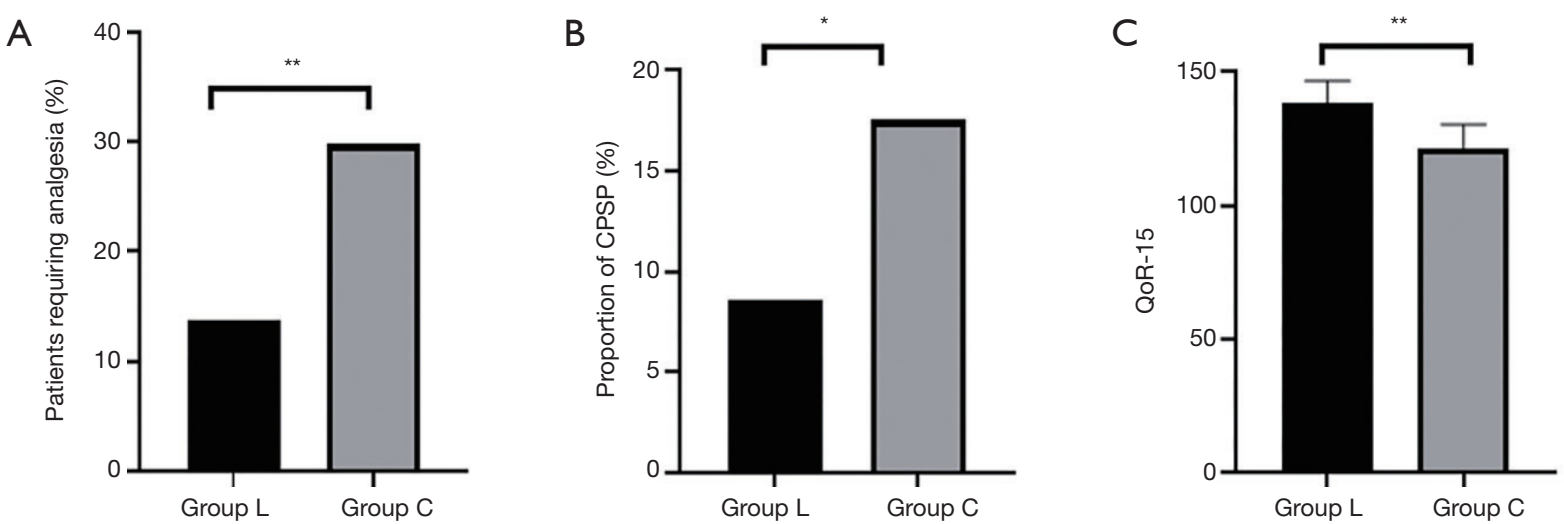

Figure 3 Comparison of the percentage of patients requiring analgesia (A), proportion of chronic postoperative pain (CPSP) (B), and postoperative comfort $(\mathrm{C})$ between the 2 groups. Proportion of CPSP in the intravenous lidocaine infusion group (group L) was significantly lower than that in the group $\mathrm{C}$, and the difference was statistically significant $(\mathrm{P}<0.05)$. The percentage of patients requiring analgesia and postoperative comfort of group $\mathrm{L}$ was significantly higher than those of group $\mathrm{C}$, and the difference was statistically significant $(\mathrm{P}<0.01)$. ${ }^{*} \mathrm{P}<0.05 ;{ }^{* *} \mathrm{P}<0.01$. QoR-15, Quality of Recovery Score-15.
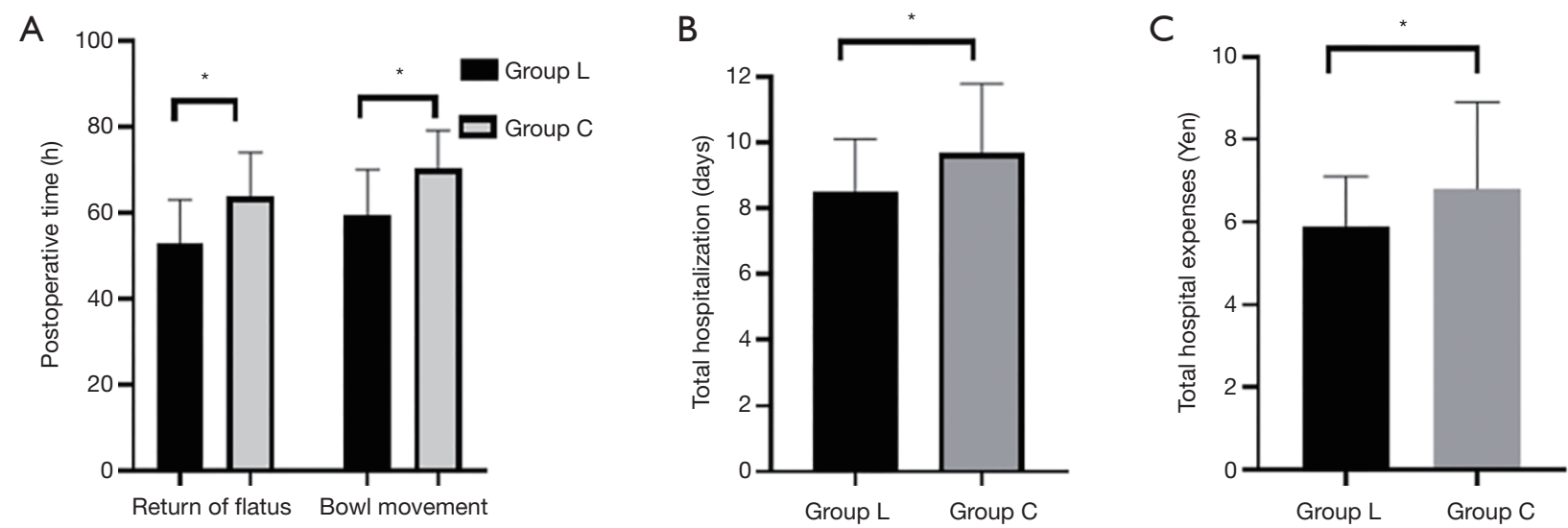

Figure 4 Comparison of postoperative gastrointestinal function recovery time (A), hospitalization time (B), and hospitalization expenses (C) between the 2 groups. Compared with the control group (group C), the return of flatus, bowl movement, the hospitalization times, and the hospitalization expenses of the intravenous lidocaine infusion group (group L) were statistically significant $(\mathrm{P}<0.05)$. ${ }^{*} \mathrm{P}<0.05$.

which was beneficial to patients' long-term quality of life after surgery. At present, controversy still exists on the mechanism of action, effective dose range, effective time, and other aspects related to intravenous lidocaine analgesia and warrant further in-depth study. Previously published studies have shown that intravenous lidocaine infusion inhibits the occurrence of CPSP and could be related to lidocaine's reduction of central sensitization, inhibition of central nervous system inflammation, inhibition of $\mathrm{Na}$ channel expression, and ectopic nerve discharge (12). In addition, some studies have indicated that lidocaine may inhibit the regulate of transient receptor potential cation channel (TRPV) 6, thereby reducing tumor cell invasion and migration, inhibiting tumor metastasis or recurrence, and reducing the occurrence of cancer pain (13). In terms of dosage, Abelson et al. found that after intravenous bolus of 1-2 $\mathrm{mg} / \mathrm{kg}$ lidocaine, regardless of whether the intravenous infusion of $1.5 \mathrm{mg} \cdot \mathrm{kg}^{-1} \cdot \mathrm{h}^{-1}$ was continued, the corresponding plasma concentration was maintained at about $2 \mu \mathrm{g} / \mathrm{mL}$, much lower than the plasma concentration of $5 \mu \mathrm{g} / \mathrm{mL}$, which usually causes the toxic effects of lidocaine (14). The dose of continuous lidocaine used in the present study was also the dose range that was considered suitable for people in the existing literature (13). 
The evaluation of patients' postoperative comfort is linked to ERAS. In 2018, Myles et al. published an expert consensus on standardized outcome indicators for perioperative patient comfort (15). The consensus recommends 6 outcome indicators related to patient comfort as follows: pain, nausea, vomiting, gastrointestinal recovery, activity status, sleep quality, and postoperative recovery quality. The QoR-15 is an index that is widely used to evaluate postoperative recovery, including comfort (16). The proportion of POI after gastrointestinal tumor surgery is a common complication, and cause postoperative nausea, vomiting, abdominal pain, abdominal distension, and delayed defecation, causing discomfort and abdominal pain. The mechanism of POI is complex, including surgical trauma, inflammation, anesthetic drugs, nerve dysfunction and other factors. Therefore, reducing the incidence of POI is an important index to evaluate the postoperative recovery of such patients. The current studies related to ERAS in gastrointestinal tumor surgery have indicated that early resumption of eating can reduce the incidence of POI and infection after gastrointestinal tumor surgery, and shorten the length of hospitalization (17). However, some studies showed the opposite opinion (18). It has been reported in the literature that the specific mechanism of intravenous lidocaine infusion is significantly reduce the production of complement and pro-inflammatory cytokines interleukin (IL)-8 and IL-6, thereby inhibiting gastrointestinal inflammatory reactions and reducing the occurrence of POI, and directly inhibit the mesenteric sympathetic plexus, reduce the consumption of opioids after surgery $(19,20)$. In the present study, the first return of flatus and bowl movement recommended by the guidelines was used to objectively evaluate the patients' gastrointestinal function recovery. It was found that intravenous lidocaine infusion significantly improved the postoperative recovery of patients in group L compared with patients in group C, including the first return of flatus and bowl movement after the operation. It was also observed that intravenous lidocaine infusion can accelerate patients' postoperative recovery, improve their postoperative comfort, and reduce the length of hospitalization and expenses, in line with ERAS concepts.

Two patients in group $\mathrm{L}$ and 3 patients in group $\mathrm{C}$ withdrew due to postoperative follow-up failure, underwent multiple surgeries, or transferred to ICU after surgery. These patients' data were excluded in the analysis in order to reduce systematic bias. Intravenous lidocaine infusion has the advantages of reducing postoperative pain, reducing inflammatory reaction, reducing the use of analgesics, but also has some disadvantages. The most common adverse events of intravenous lidocaine during awake patients are neuropsychiatric symptoms. Seven patients complained of drowsiness and 3 patients complained of dry mouth in group $\mathrm{L}$ and 2 patients complained of dizziness, 5 patients of drowsiness, and 1 patient of nausea and vomiting in group C. No serious adverse events associated with lidocaine were found in either group. Therefore, the uniform standard for intravenous lidocaine for different types of surgeries is typical necessary. The number of cases in the present study was limited, and larger clinical studies are needed to formulate a standard medication guide plan for intravenous lidocaine infusion for different types of surgeries.

The present study has some limitations. First, the findings showed that the intraoperative use of lidocaine can significantly reduce postoperative pain, but the total amount of opioid analgesics during operation was not counted. Second, only one dose of intravenous lidocaine infusion has observed in this study, and the minimum effective dosage of intravenous lidocaine infusion in gastrointestinal tumor surgery is not known. Third, the concentration of lidocaine in patient plasma was monitored. Finally, we only used the NRS to assess the incidence of chronic pain at 3 months postoperatively and did not perform a follow-up assessment of the long-term quality of life or survival rate of patients after surgery. A larger sample of clinical studies will be used in our follow-up study to explore in depth the effective dose range of intravenous lidocaine infusion for patient comfort perioperatively.

\section{Conclusions}

In summary, the continuous intravenous lidocaine infusion during gastrointestinal tumor surgery can reduce acute and chronic postoperative pain and promote postoperative gastrointestinal function recovery and enhance the perioperative comfort.

\section{Acknowledgments}

The authors wish to thank Liuliu for assistance with statistical analysis and Jiang Lu, Li Jiachen, Yang Di, Yang Mengchang, Ma Ding, Lin Dayong, Jin Gang for operational assistance. We also thank Rosie Scott, $\mathrm{PhD}$, for editing the English text of a draft of this manuscript.

Funding: None. 


\section{Footnote}

Reporting Checklist: The authors have completed the CONSORT reporting checklist. Available at http://dx.doi. org/10.21037/jgo-20-505

Data Sharing Statement: Available at http://dx.doi. org/10.21037/jgo-20-505

Conflicts of Interest: All authors have completed the ICMJE uniform disclosure form (available at http://dx.doi. org/10.21037/jgo-20-505). The authors have no conflicts of interest to declare.

Ethical Statement: The authors are accountable for all aspects of the work in ensuring that questions related to the accuracy or integrity of any part of the work are appropriately investigated and resolved. The present study was a randomized, double-blind, controlled study. It was reviewed and approved by the Medical Ethics Committee of Sichuan Provincial People's Hospital. All procedures performed in this study involving human participants were in accordance with the Declaration of Helsinki (as revised in 2013). Written informed consent was obtained from patients prior to their participation.

Open Access Statement: This is an Open Access article distributed in accordance with the Creative Commons Attribution-NonCommercial-NoDerivs 4.0 International License (CC BY-NC-ND 4.0), which permits the noncommercial replication and distribution of the article with the strict proviso that no changes or edits are made and the original work is properly cited (including links to both the formal publication through the relevant DOI and the license). See: https://creativecommons.org/licenses/by-nc-nd/4.0/.

\section{References}

1. Cats A, Jansen EPM, van Grieken NCT, et al. Chemotherapy versus chemoradiotherapy after surgery and preoperative chemotherapy for resectable gastric cancer (CRITICS): an international, open-label, randomised phase 3 trial. Lancet Oncol 2018;19:616-28.

2. Venara A, Neunlist M, Slim K, et al. Postoperative ileus: pathophysiology, incidence, and prevention. J Visc Surg 2016;153:439-46.

3. Minarich MJ, Schwarz RE. Experience with a simplified feeding jejunostomy technique for enteral nutrition following major visceral operations. Transl Gastroenterol Hepatol 2018;3:44.

4. Soto G, Naranjo González M, Calero F. Intravenous lidocaine infusion. Perfusión de lidocaína intravenosa. Rev Esp Anestesiol Reanim 2018;65:269-74.

5. McCarthy GC, Megalla SA, Habib AS. Impact of intravenous lidocaine infusion on postoperative analgesia and recovery from surgery: a systematic review of randomized controlled trials. Drugs 2010;70:1149-63.

6. Kranke P, Jokinen J, Pace NL, et al. Continuous intravenous perioperative lidocaine infusion for postoperative pain and recovery. Cochrane Database Syst Rev 2015;(7):CD009642.

7. Tikhonov DB, Zhorov BS. Mechanism of sodium channel block by local anesthetics, antiarrhythmics, and anticonvulsants. J Gen Physiol 2017;149:465-81.

8. Clattenburg EJ, Nguyen A, Yoo T, et al. Intravenous Lidocaine Provides Similar Analgesia to Intravenous Morphine for Undifferentiated Severe Pain in the Emergency Department: A Pilot, Unblinded Randomized Controlled Trial. Pain Med 2019;20:834-9.

9. Liu H, Lu F, Zhou D, et al. The Analgesic and Emotional Response to Intravenous Lidocaine Infusion in the Treatment of Postherpetic Neuralgia: A Randomized, Double-Blinded, Placebo-controlled Study. Clin J Pain 2018;34:1025-31.

10. Dunn LK, Durieux ME. Perioperative Use of Intravenous Lidocaine. Anesthesiology 2017;126:729-37.

11. Weibel S, Jokinen J, Pace NL, et al. Efficacy and safety of intravenous lidocaine for postoperative analgesia and recovery after surgery: a systematic review with trial sequential analysis. Br J Anaesth 2016;116:770-83.

12. Soto G, Naranjo González M, Calero F. Intravenous lidocaine infusion. Perfusión de lidocaína intravenosa. Rev Esp Anestesiol Reanim 2018;65:269-74.

13. Lee JT, Sanderson CR, Xuan W, et al. Lidocaine for Cancer Pain in Adults: A Systematic Review and MetaAnalysis. J Palliat Med 2019;22:326-34.

14. Abelson KS, Höglund AU. Intravenously administered lidocaine in therapeutic doses increases the intraspinal release of acetylcholine in rats. Neurosci Lett 2002;317:93-6.

15. Myles PS, Boney O, Botti M, et al. Systematic review and consensus definitions for the Standardised Endpoints in Perioperative Medicine (StEP) initiative: patient comfort. Br J Anaesth 2018;120:705-11.

16. Kleif J, Waage J, Christensen KB, et al. Systematic review of the QoR-15 score, a patient- reported outcome 
measure measuring quality of recovery after surgery and anaesthesia. Br J Anaesth 2018;120:28-36.

17. Pędziwiatr M, Mavrikis J, Witowski J, et al. Current status of enhanced recovery after surgery (ERAS) protocol in gastrointestinal tumor surgery. Med Oncol 2018;35:95.

18. Barclay KL, Zhu YY, Tacey MA. Nausea, vomiting and return of bowel function after colorectal surgery. ANZ J Surg 2015;85:823-8.

19. Weibel S, Jelting Y, Pace NL, et al. Continuous intravenous perioperative lidocaine infusion for postoperative pain and recovery in adults. Cochrane Database Syst Rev 2018;6:CD009642.

20. Cooke C, Kennedy ED, Foo I, et al. Meta-analysis of the effect of perioperative intravenous lidocaine on return of gastrointestinal function after colorectal surgery. Tech Coloproctol 2019;23:15-24.

(English Language Editor: R. Scott)
Cite this article as: Dai Y, Jiang R, Su W, Wang M, Liu Y, Zuo Y. Impact of perioperative intravenous lidocaine infusion on postoperative pain and rapid recovery of patients undergoing gastrointestinal tumor surgery: a randomized, double-blind trial. J Gastrointest Oncol 2020;11(6):1274-1282. doi: 10.21037/jgo20-505 